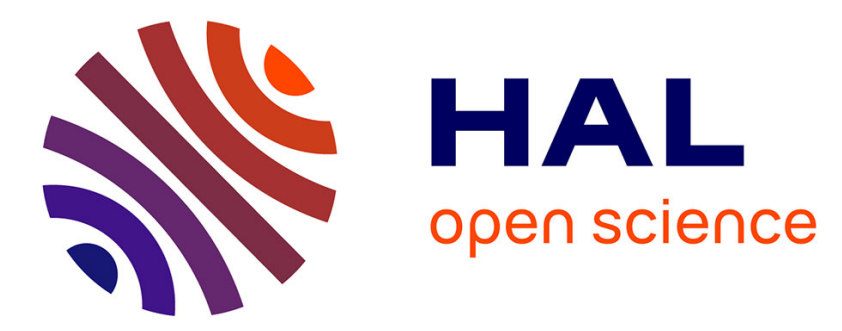

\title{
Near-field radiative heat transfer between twisted nanoparticle gratings
}

\author{
Minggang Luo, Junming Zhao, Mauro Antezza
}

\section{To cite this version:}

Minggang Luo, Junming Zhao, Mauro Antezza. Near-field radiative heat transfer between twisted nanoparticle gratings. Applied Physics Letters, 2020, 117, pp.053901. 10.1063/5.0018329 . hal02912756

\section{HAL Id: hal-02912756 \\ https://hal.science/hal-02912756}

Submitted on 14 Oct 2020

HAL is a multi-disciplinary open access archive for the deposit and dissemination of scientific research documents, whether they are published or not. The documents may come from teaching and research institutions in France or abroad, or from public or private research centers.
L'archive ouverte pluridisciplinaire HAL, est destinée au dépôt et à la diffusion de documents scientifiques de niveau recherche, publiés ou non, émanant des établissements d'enseignement et de recherche français ou étrangers, des laboratoires publics ou privés. 


\section{Near-field radiative heat transfer between twisted nanoparticle gratings}

Cite as: Appl. Phys. Lett. 117, 053901 (2020); https://doi.org/10.1063/5.0018329

Submitted: 13 June 2020 . Accepted: 27 July 2020 . Published Online: 06 August 2020

Minggang Luo (D), Junming Zhao, and Mauro Antezza (D)
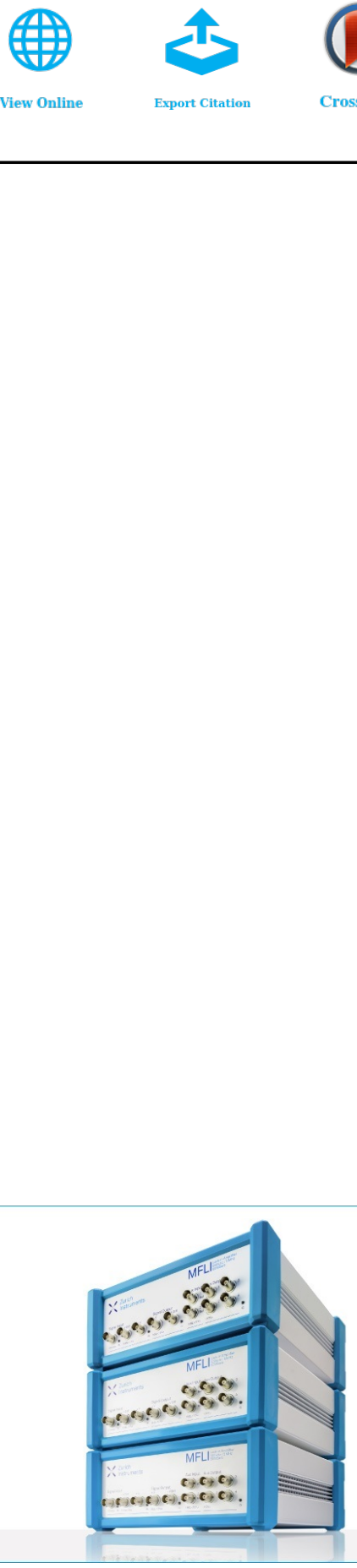


\title{
Near-field radiative heat transfer between twisted nanoparticle gratings
}

\author{
Cite as: Appl. Phys. Lett. 117, 053901 (2020); doi: 10.1063/5.0018329 \\ Submitted: 13 June 2020 . Accepted: 27 July 2020 . \\ Published Online: 6 August 2020
}

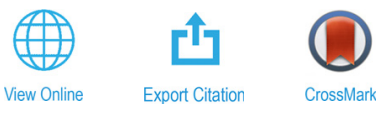

Minggang Luo, ${ }^{1,2}$ (D) Junming Zhao, ${ }^{1,3, a)}$ and Mauro Antezza ${ }^{2,4, b)}$ (D)

\begin{abstract}
AFFILIATIONS
1School of Energy Science and Engineering, Harbin Institute of Technology, 92 West Street, Harbin 150001, China

${ }^{2}$ Laboratoire Charles Coulomb (L2C) UMR 5221 CNRS-Université de Montpellier, F-34095 Montpellier, France

${ }^{3}$ Key Laboratory of Aerospace Thermophysics, Ministry of Industry and Information Technology, Harbin 150001, China

${ }^{4}$ Institut Universitairé de France, 1 rue Descartes, F-75231 Paris Cedex 05, France
\end{abstract}

a)jmzhao@hit.edu.ch

b) Author to whom correspondence should be addressed: mauro.antezza@umontpellier.fr

\begin{abstract}
We study the near-field radiative heat transfer between two twisted finite-size polar dielectric nanoparticle gratings. Different from previous studies of the same configuration, we do not rely on any approximated effective medium theory to describe the gratings. By the full many-body radiative heat transfer theory, we are able to investigate how the size, distance, and relative orientation between the gratings influence the radiative heat flux. By changing the twisting angle $\theta$, we show a significant oscillation of the thermal conductance $G(\theta)$, due to the size effect for gratings of both square and circular shapes. The distance- and twisting-dependent coupling between the gratings accounts for a strong and characteristic modulation of radiative thermal conductance with implications for the energy management, sensing, and the micro-electromechanical system (MEMS) and nano-electromechanical system (NEMS) devices.
\end{abstract}

Published under license by AIP Publishing. https://doi.org/10.1063/5.0018329

Near-field radiative heat transfer (NFRHT) has recently attracted much attention for both fundamental and applicative reasons. The radiative heat exchange between two objects with a separation distance comparable to or less than the thermal wavelength $\lambda_{T}=\hbar c / k_{B} T$ can exceed by several orders of magnitude the Planckian blackbody limit, which has been theoretically investigated for physical systems of different geometries (e.g., two planar surfaces, ${ }^{1-6}$ two isolated nanoparticles, ${ }^{7-9}$ two spheres, ${ }^{10}$ one dipole and surface, ${ }^{11}$ two nanoparticles above a substrate, ${ }^{12-15}$ and two nanoparticles separated by a multilayer plate $^{16}$ ), and has also been proved experimentally recently (e.g., two plates, ${ }^{17-21}$ one plate, and one sphere or tip ${ }^{22-24}$ ).

Active tuning of NFRHT is of great interest and importance for micro-nanoscale heat management. To this purpose, several proposals investigated the NFRHT between twisted gratings, ${ }^{25}$ possibly realized with graphene-coated strips, ${ }^{26}$ or using natural anisotropic materials (two multiple black phosphorus layers) with a twisting relative angle between the upper and lower multiple layers. ${ }^{27}$ Some other important progress has also been reported in tuning and manipulating the NFRHT on the micro-nanoscale, e.g., pattern-free thermal modulator, ${ }^{28}$ strain-induced modulation, ${ }^{29}$ thermal routing, ${ }^{30}$ thermal Hall effect, ${ }^{31}$ and heat flux splitter, ${ }^{32}$ to name a few.
NFRHT modulations via twisted gratings typically use the effective medium approximation (EMA) $)^{33-35}$ to describe the gratings, and consider the approximation of infinite-size systems. ${ }^{25}$ When the distance between two parallel gratings is comparable to or less than the grating periods, the EMA theory cannot be used. In addition, the finite size effect will also bring new challenges when using the EMA to calculate NFRHT between two finite gratings. For the finite size physical system, as compared to the corresponding infinite system, some new physical insight may be introduced. This has been recently shown when considering the Casimir torque between two finite gratings at different twisting angles, where the torque per area can reach extremely large values, increasing without bounds with the size of the system. ${ }^{36}$ An investigation of finite-size and a beyond-EMA approximation effects between twisted gratings is still missing for the NFRHT.

In this Letter, by investigating the NFRHT between two finitesize polar dielectric nanoparticle gratings with a relative twisting angle $\theta$ (see Fig. 1) and by using a full many-body radiative heat transfer theory, ${ }^{37}$ we show that finite-size effects and an exact calculation beyond the EMA theory lead to new qualitative and quantitative effects and features. 


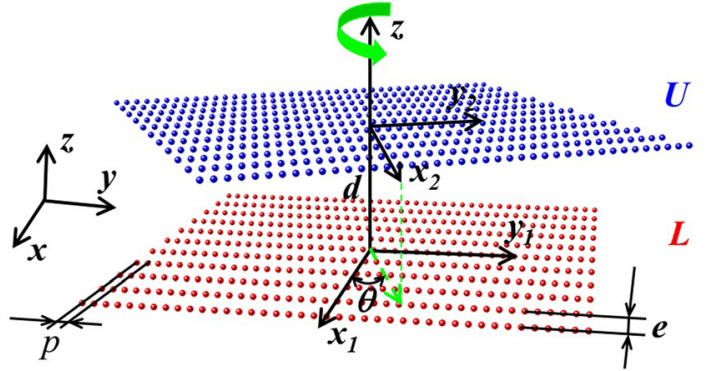

FIG. 1. Two nanoparticle gratings ( $L$ and $U)$, realized with parallel nanoparticle chains, are separated by a distance $d$ and twisted by an angle $\theta$. The grating lattice period is $e$, while $p$ is the distance between neighboring nanoparticles in each nanoparticle chain.

As shown in Fig. 1, each ensemble is composed of many nanoparticle chains. When calculating the radiative thermal conductance $G$, we consider that the two ensembles are near the thermal equilibrium around the temperature $T$. Inside each nanoparticle chain, $p$ is the distance between neighboring nanoparticles, while $e$ is the separation distance between two neighboring chains. Nanoparticle radius $a$ is fixed as the smallest length-scale in the problem, allowing for the dipole approximation. ${ }^{38,39}$ The separation distance between the gratings $\mathrm{L}$ and $\mathrm{U}$ center to center is $d$. The minimum nanoparticle separation in each chain is set to $p=3 a$. When considering heat exchange between such nanoparticle ensembles where $e>3 p$, each ensemble behaves like a grating. In addition, the effects of the coupling between the two gratings, breaking of symmetry, and collective many-body interaction on NFRHT between the two nanoparticle gratings are fully taken into account by the many-body radiative heat transfer theory. ${ }^{39-44}$

We focus on the radiative thermal conductance $G(\theta)$ between two polar dielectric $\mathrm{SiC}$ nanoparticle gratings with a relative angle $\theta$, which is the sum of that between all possible nanoparticle pairs (one from the grating $\mathrm{L}$ and the other one from grating $\mathrm{U}$ ) and is defined as follows: ${ }^{45}$

$$
G(\theta)=\sum_{\mathrm{i} \in \mathrm{U}} \sum_{\mathrm{j} \in \mathrm{L}} G_{\mathrm{ij}},
$$

where $G_{\mathrm{ij}}$ is the thermal conductance between two arbitrary nanoparticles $i$ and $j$ and yields

$$
G_{\mathrm{ij}}=3 \int_{0}^{+\infty} \frac{\mathrm{d} \omega}{2 \pi} \frac{\partial \Theta(\omega, T)}{\partial T} \mathscr{T}_{\mathrm{i}, \mathrm{j}}(\omega),
$$

where $\omega$ is the angular frequency, $\Theta(\omega, T)$ is the mean energy of the Planck's oscillator, and transmission coefficient $\mathscr{T}_{i, j}(\omega)$ between the jth and ith dielectric particles is given as follows: $:^{37,39}$

$$
\mathscr{T}_{\mathrm{i}, \mathrm{j}}(\omega)=\frac{4}{3} k^{4} \operatorname{Im}\left(\chi_{E}^{\mathrm{i}}\right) \operatorname{Im}\left(\chi_{E}^{\mathrm{j}}\right) \operatorname{Tr}\left(G_{\mathrm{ij}}^{E E} G_{\mathrm{ij}}^{E E^{\dagger}}\right),
$$

where the parameter $\chi_{E}^{\text {ior } j}=\alpha_{E}^{\text {ior } j}-\frac{i k^{3}}{6 \pi}\left|\alpha_{E}^{\text {i or j }}\right|^{2}$ is introduced, ${ }^{8} \alpha_{E}^{\text {i or j }}$ is the corresponding electric dipole polarizability described as $4 \pi a^{3}(\varepsilon-1) /(\varepsilon+2)$ in the Clausius-Mossotti form, ${ }^{37}$ the permittivity for the polar dielectric $\mathrm{SiC}$ is described by the Drude-Lorentz model ${ }^{46}$ $\epsilon(\omega)=\epsilon_{\infty}\left(\omega^{2}-\omega_{l}^{2}+i \gamma \omega\right) /\left(\omega^{2}-\omega_{t}^{2}+i \gamma \omega\right)$, with parameters $\epsilon_{\infty}=6.7, \omega_{l}=1.827 \times 10^{14} \mathrm{rad} \cdot \mathrm{s}^{-1}, \omega_{t}=1.495 \times 10^{14} \mathrm{rad} \cdot \mathrm{s}^{-1}$, and $\gamma=0.9 \times 10^{12} \mathrm{rad} \cdot \mathrm{s}^{-1}, k$ is the vacuum wavevector and $G_{\mathrm{ij}}^{E E}$ is the electric-electric Green's function in the particulate system considering many-body interaction, which is the element of the following left matrix,

$\left(\begin{array}{cccc}0 & G_{12}^{E E} & \cdots & G_{1 N}^{E E} \\ G_{21}^{E E} & 0 & \ddots & \vdots \\ \vdots & \vdots & \ddots & G_{(N-1) N}^{E E} \\ G_{N 1}^{E E} & G_{N 2}^{E E} & \cdots & 0\end{array}\right) \mathbb{A}=\left(\begin{array}{cccc}0 & G_{0,12}^{E E} & \cdots & G_{0,1 N}^{E E} \\ G_{0,21}^{E E} & 0 & \ddots & \vdots \\ \vdots & \vdots & \ddots & G_{0,(N-1) N}^{E E} \\ G_{0, N 1}^{E E} & G_{0, N 2}^{E E} & \cdots & 0\end{array}\right)$

where $G_{0, \mathrm{ij}}^{E E}=\frac{e^{i k r}}{4 \pi r}\left[\left(1+\frac{i k r-1}{k^{2} r^{2}}\right) \mathbb{I}_{3}+\frac{3-3 i k r-k^{2} r^{2}}{k^{2} r^{2}} \hat{\mathbf{r}} \otimes \hat{\mathbf{r}}\right]$ is the free space Green's function connecting two nanoparticles at $\mathbf{r}_{i}$ and $\mathbf{r}_{j}, r$ is the magnitude of the separation vector $\mathbf{r}=\mathbf{r}_{i}-\mathbf{r}_{j}, \hat{\mathbf{r}}$ is the unit vector $\mathbf{r} / r, \mathbb{I}_{3}$ is a $3 \times 3$ identity matrix, and the matrix $\mathbb{A}$ including manybody interaction is defined as

$$
\mathbb{A}=\mathbb{I}_{3 N}-k^{2}\left(\begin{array}{cccc}
0 & \alpha_{E}^{1} G_{0,12}^{E E} & \cdots & \alpha_{E}^{1} G_{0,1 N}^{E E} \\
\alpha_{E}^{2} G_{0,21}^{E E} & 0 & \ddots & \vdots \\
\vdots & \vdots & \ddots & \alpha_{E}^{N-1} G_{0,(N-1) N}^{E E} \\
\alpha_{E}^{N} G_{0, N 1}^{E E} & \cdots & \alpha_{E}^{N} G_{0, N(N-1)}^{E E} & 0
\end{array}\right) \text {, }
$$

where $\mathbb{I}_{3 N}$ is a $3 N \times 3 N$ identity matrix.

Now, we discuss the numerical results using above expressions for NFRHT between two polar dielectric SiC nanoparticle gratings. In Fig. 2, we show the scaled thermal conductance $G(\theta) / N$ as a function of the twisting angle $\theta$. Both square gratings and circular gratings are considered, of which the schematics are shown in the inset of Fig. 2. Three lateral sizes are considered, $D_{1}=0.8 \mu \mathrm{m}, D_{2}=1.4 \mu \mathrm{m}$, and $D_{3}=2.8 \mu \mathrm{m}$, respectively. $a=20 \mathrm{~nm}, \quad T=300 \mathrm{~K}, d=80 \mathrm{~nm}$, $p=60 \mathrm{~nm}$, and $e=200 \mathrm{~nm}$.

For both the square and circular gratings, with increasing twisting angles, $G(\theta)$ decreases to its first valley and then oscillates slightly. The twisting angle corresponding to the first valley of $G(\theta)$ is $\theta_{\mathrm{m}}$. For the circular gratings of three lateral lengths $\left(D_{1}, D_{2}\right.$, and $\left.D_{3}\right), \theta_{\mathrm{m}}$ values are $24^{\circ}, 10^{\circ}$, and $6.8^{\circ}$, respectively. $\theta_{\mathrm{m}}$ increases with the decreasing lateral length of the gratings. The amplitude of the $G(\theta)$ oscillation corresponding to $D_{1}$ is much larger than that corresponding to $D_{2}$ and $D_{3}$. As the lateral length of the circular grating increases, the amplitude of the oscillation of $G(\theta)$ decreases gradually. The thermal conductance $G(\theta)$ as a function of $\theta$ tends to converge to a given curve when the size of gratings increases. The size effect accounts for the oscillating behavior of $G(\theta)$ and was absent in previous studies using infinite-systems and EMA approximations. It is worth stressing that an oscillation of the thermal conductance due to a lateral shift of two aligned gratings has been recently reported. ${ }^{45}$

Even for the same value of size $D$, the curves of $G(\theta)$ are quite different if we consider square or circular gratings. In particular, for large $\theta\left(50^{\circ}-90^{\circ}\right), G(\theta)$ increases monotonically with $\theta$ for the square gratings, while the oscillations are still present for circular gratings. 


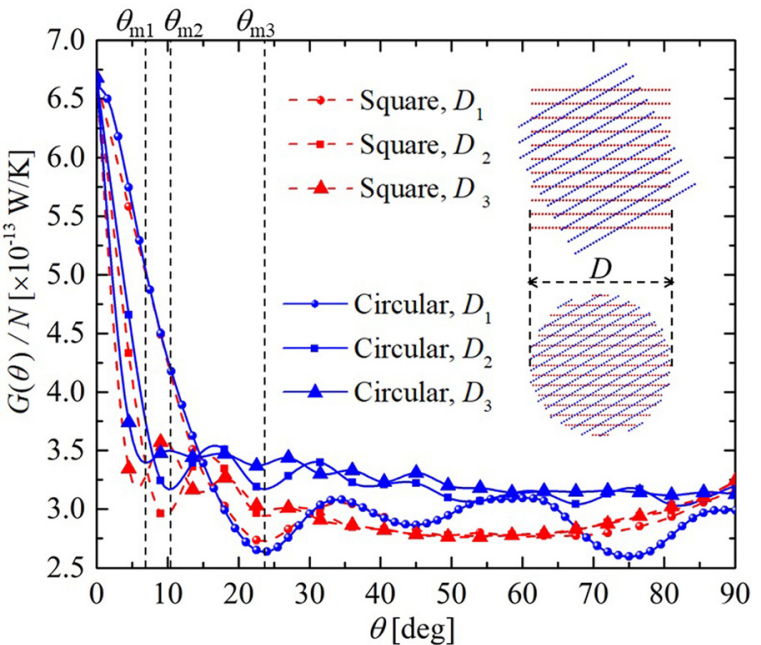

FIG. 2. The scaled thermal conductance $G(\theta) / N$ as a function of the twisting angle $\theta$. A scheme for the square and circular grating configurations is shown in the inset. Three different lateral lengths are considered, $D_{1}=0.8 \mu \mathrm{m}, D_{2}=1.4 \mu \mathrm{m}$, and $D_{3}=2.8 \mu \mathrm{m}$, respectively. $d=80 \mathrm{~nm}, a=20 \mathrm{~nm}, T=300 \mathrm{~K}, p=60 \mathrm{~nm}$, and $e=10 \mathrm{a}=200 \mathrm{~nm}$. For the circular grating configuration, $\theta_{\mathrm{m} 1}, \theta_{\mathrm{m} 2}$, and $\theta_{\mathrm{m} 3}$ correspond to the angle where $G(\theta)$ decreases to its first valley value, respectively, for systems having lateral lengths $D_{1}, D_{2}$, and $D_{3}$.

Hence, we deduce that the overlap between the $\mathrm{L}$ and $\mathrm{U}$ gratings, which is different in the case of square and circular configurations, significantly influences the thermal conductance between two 2D ensembles, as it happens for the Casimir torque. ${ }^{36}$ At large angles, the overlap area of the two square gratings increases monotonically with the increasing twisting angle $\theta$, which may account for the monotonically increasing dependence of $G(\theta)$ on the twisting angle $\theta$. For circular gratings, the overlap area will not vary with increasing $\theta$ and the angle-dependent coupling accounts for the oscillation of $G(\theta)$ with $\theta$.

We move here to the study of the separation $d$ dependence of the thermal conductance all along the twisting. The ratio of the thermal conductance $G(\theta)$ to the thermal conductance $G\left(\theta=0^{\circ}\right)$ is shown as a function of the twisting angle $\theta$ and for different values of $d$ in Fig. 3 . Circular gratings with $D=1.8 \mu \mathrm{m}$ are considered here.

The decreasing behavior of $G(\theta) / G\left(\theta=0^{\circ}\right)$ with the twisting angle $\theta$ is significantly dependent on the distance. For the smallest distance $d=80 \mathrm{~nm}, G(\theta) / G\left(\theta=0^{\circ}\right)$ decreases from 1 to 0.47 and then oscillates in a pronounced way with increasing angles. As $d$ increases, the oscillations of $G(\theta) / G\left(\theta=0^{\circ}\right)$ with increasing $\theta$ decrease gradually. Particularly, for a large $d, G(\theta) / G\left(0^{\circ}\right)$ decreases with $\theta$ monotonically and smoothly, which is similar to the monotonic and smooth decreasing dependence of near-field heat transfer coefficient $h / h_{\max }\left(\mathrm{W} / \mathrm{m}^{2}\right)$ with the twisting angle observed for the $1 \mathrm{D}$ semi-infinite gratings. ${ }^{47,48}$ For a large $d, G(\theta) / G\left(\theta=0^{\circ}\right)$ varies in a much shorter range (e.g., for $d=200 \mathrm{~nm}$, it ranges from 1.0 to 0.8 ) as compared to the case with smaller separations (e.g., for $d=80 \mathrm{~nm}$, it ranges from 1.0 to 0.47 ). It is worthwhile to mention that the first-valley $\theta_{\mathrm{m}}$ is independent of the separation distance $d$ between the two gratings and is significantly dependent on the size of the gratings.

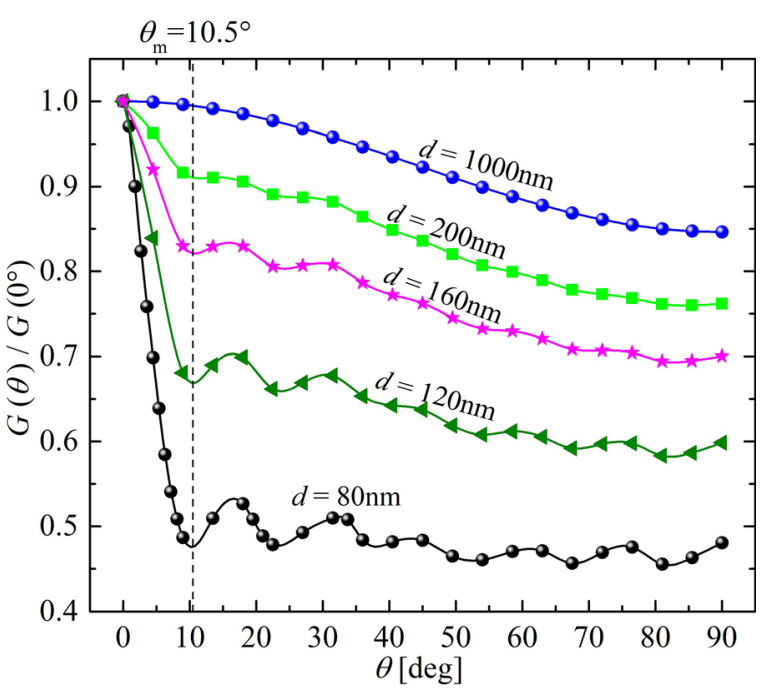

FIG. 3. The ratio $G(\theta) / G\left(\theta=0^{\circ}\right)$ for circular gratings. Five different separations $d$ are considered, $d=80 \mathrm{~nm}, 120 \mathrm{~nm}, 160 \mathrm{~nm}, 200 \mathrm{~nm}$, and $1000 \mathrm{~nm}$, respectively. $a=20 \mathrm{~nm}, T=300 \mathrm{~K}, p=60 \mathrm{~nm}, e=10 \mathrm{a}=200 \mathrm{~nm}$, and $D=1.8 \mu \mathrm{m}$.

The vacuum filling fraction $f=(e-2 a) / e$ is another parameter that can significantly influence the NFRHT. The modulation rate $\left[G\left(0^{\circ}\right)-G\left(90^{\circ}\right)\right] / G\left(0^{\circ}\right)$ is shown in Fig. 4 as a function of $f . p=60 \mathrm{~nm}$. Two different distances $d=80 \mathrm{~nm}$ and $d=200 \mathrm{~nm}$ are considered.

For the two different separations, the modulation rate $\left[G\left(0^{\circ}\right)-G\left(90^{\circ}\right)\right] / G\left(0^{\circ}\right)$ increases with the vacuum filling fraction $f$. The modulation rate for a small distance $d=80 \mathrm{~nm}$ is always much larger than that of the case with a big distance $d=200 \mathrm{~nm}$ because of a stronger coupling at small separations. The coupling between the two gratings with the twisting angle is distance dependent.

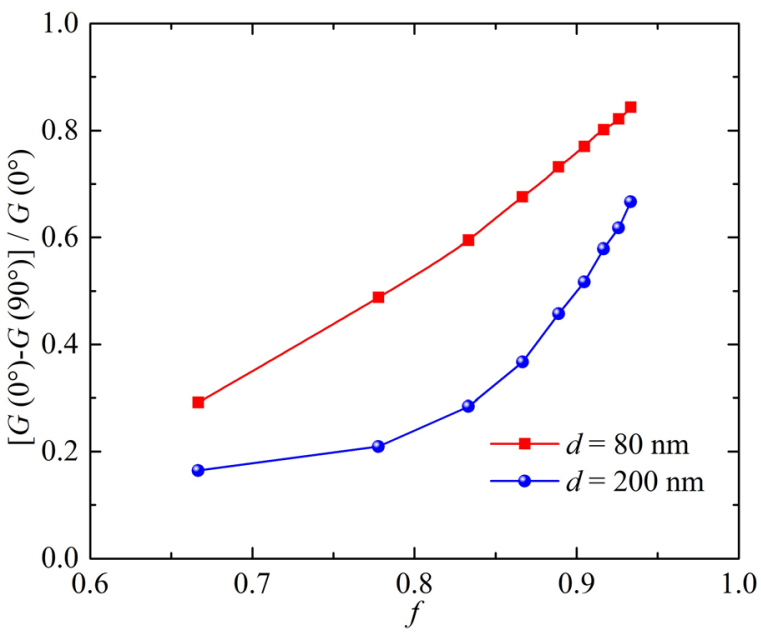

FIG. 4. $\left[G\left(0^{\circ}\right)-G\left(90^{\circ}\right)\right] / G\left(0^{\circ}\right)$ as a function of the vacuum filling fraction $f$ at two different distances $d=80 \mathrm{~nm}$ and $d=200 \mathrm{~nm}$ and for circular gratings. $T=300 \mathrm{~K}, a=20 \mathrm{~nm}, p=60 \mathrm{~nm}$, and $D=4 \mu \mathrm{m}$. 
In summary, the modulation of NFRHT between nanoparticle gratings by twisting is analyzed beyond the EMA and infinite-size approximations, which is needed for realistic experimental analysis. We studied different finite-size grating shapes (square and circular), which present remarkably different features. When twisting one grating, characteristic oscillations of the thermal conductance $G(\theta)$ are observed (more pronounced for circular gratings) and fully due to finite-size and beyond-EMA effects. $G(\theta)$ converges to a given shape with the increasing size of gratings. In addition, the modulation rate $\left[G\left(0^{\circ}\right)-G\left(90^{\circ}\right)\right] / G\left(0^{\circ}\right)$ is significantly dependent on the vacuum filling fraction. This work may help for energy management at the nanoscale with relevant implications for the micro-electromechanical system (MEMS) and nano-electromechanical system (NEMS) devices.

As for the infinite system, most recently, the Fourier modal method was raised for the description of nanoparticle lattices in the dipole approximation, ${ }^{49,50}$ which makes the direct investigation on NFRHT between infinite periodic nanoparticle lattices possible.

The support of this work by the National Natural Science Foundation of China (No. 51976045) is gratefully acknowledged. M.A. acknowledges support from the Institute Universitairé de France, Paris, France (UE). M.G.L. is also thankful for the support from the China Scholarship Council (No. 201906120208).

\section{DATA AVAILABILITY}

The data that support the findings of this study are available from the corresponding author upon reasonable request.

\section{REFERENCES}

${ }^{1}$ S. M. Rytov, Y. A. Kravtsov, and V. I. Tatarskii, Priniciples of Statistical Radiophysics (Springer-Verlag, 1989), Vol. 3.

${ }^{2}$ D. Polder and M. Van Hove, "Theory of radiative heat transfer between closely spaced bodies," Phys. Rev. B 4, 3303-3314 (1971)

${ }^{3}$ J. J. Loomis and H. J. Maris, "Theory of heat transfer by evanescent electromagnetic waves," Phys. Rev. B 50, 18517-18524 (1994).

${ }^{4}$ R. Carminati and J.-J. Greffet, "Near-field effects in spatial coherence of thermal sources,” Phys. Rev. Lett. 82, 1660-1663 (1999).

${ }^{5}$ A. V. Shchegrov, K. Joulain, R. Carminati, and J.-J. Greffet, "Near-field spectral effects due to electromagnetic surface excitations," Phys. Rev. Lett. 85, 1548-1551 (2000).

${ }^{6}$ A. I. Volokitin and B. N. J. Persson, "Radiative heat transfer between nanostructures," Phys. Rev. B 63, 205404 (2001).

${ }^{7}$ P. O. Chapuis, M. Laroche, S. Volz, and J.-J. Greffet, "Radiative heat transfer between metallic nanoparticles,” Appl. Phys. Lett. 92, 201906 (2008).

${ }^{8}$ A. Manjavacas and F. J. García de Abajo, "Radiative heat transfer between neighboring particles,” Phys. Rev. B 86, 075466 (2012).

${ }^{9}$ M. Nikbakht, "Radiative heat transfer between core-shell nanoparticles," J. Quant. Spectrosc. Radiat. Transfer 221, 164-171 (2018).

${ }^{10}$ A. Narayanaswamy and G. Chen, "Thermal near-field radiative transfer between two spheres," Phys. Rev. B 77, 075125 (2008).

${ }^{11}$ P. O. Chapuis, M. Laroche, S. Volz, and J.-J. Greffet, "Near-field induction heating of metallic nanoparticles due to infrared magnetic dipole contribution," Phys. Rev. B 77, 125402 (2008).

${ }^{12}$ R. Messina, S.-A. Biehs, and P. Ben-Abdallah, "Surface-mode-assisted amplification of radiative heat transfer between nanoparticles," Phys. Rev. B 97, 165437 (2018).

${ }^{13}$ J. Dong, J. M. Zhao, and L. H. Liu, "Long-distance near-field energy transport via propagating surface waves," Phys. Rev. B 97, 075422 (2018).

${ }^{14}$ Y. Zhang, M. Antezza, H. L. Yi, and H. P. Tan, "Metasurface-mediated anisotropic radiative heat transfer between nanoparticles,” Phys. Rev. B 100, 085426 (2019).

${ }^{15}$ M. J. He, H. Qi, Y. T. Ren, Y. J. Zhao, and M. Antezza, “Graphene-based thermal repeater," Appl. Phys. Lett. 115, 263101 (2019).
${ }^{16}$ Y. Zhang, H. L. Yi, H. P. Tan, and M. Antezza, "Giant resonant radiative heat transfer between nanoparticles,” Phys. Rev. B 100, 134305 (2019).

${ }^{17}$ R. S. Ottens, V. Quetschke, S. Wise, A. A. Alemi, R. Lundock, G. Mueller, D. H. Reitze, D. B. Tanner, and B. F. Whiting, "Near-field radiative heat transfer between macroscopic planar surfaces," Phys. Rev. Lett. 107, 014301 (2011).

${ }^{18}$ M. Lim, S. S. Lee, and B. J. Lee, "Near-field thermal radiation between doped silicon plates at nanoscale gaps," Phys. Rev. B 91, 195136 (2015).

${ }^{19}$ J. I. Watjen, B. Zhao, and Z. M. Zhang, "Near-field radiative heat transfer between doped-si parallel plates separated by a spacing down to $200 \mathrm{~nm}$," Appl. Phys. Lett. 109, 203112 (2016).

${ }^{20}$ M. Ghashami, H. Geng, T. Kim, N. Iacopino, S. K. Cho, and K. Park, "Precision measurement of phonon-polaritonic near-field energy transfer between macroscale planar structures under large thermal gradients," Phys. Rev. Lett. 120, 175901 (2018).

${ }^{21}$ J. DeSutter, L. Tang, and M. Francoeur, "A near-field radiative heat transfer device,” Nat. Nanotechnol. 14, 751-755 (2019).

${ }^{22}$ S. Shen, A. Narayanaswamy, and G. Chen, "Surface phonon polaritons mediated energy transfer between nanoscale gaps," Nano Lett. 9, 2909-2913 (2009).

${ }^{23}$ E. Rousseau, A. Siria, G. Jourdan, S. Volz, F. Comin, J. Chevrier, and J.-J. Greffet, "Radiative heat transfer at the nanoscale," Nat. Photonics 3, 514-517 (2009).

${ }^{24}$ B. Song, Y. Ganjeh, S. Sadat, D. Thompson, A. Fiorino, V. Fernández-Hurtado, J. Feist, F. J. Garcia-Vidal, J. C. Cuevas, P. Reddy, and E. Meyhofer, "Enhancement of near-field radiative heat transfer using polar dielectric thin films," Nat. Nanotechnol. 10, 253 (2015).

${ }^{25}$ S.-A. Biehs, F. S. S. Rosa, and P. Ben-Abdallah, "Modulation of near-field heat transfer between two gratings," Appl. Phys. Lett. 98, 243102 (2011).

${ }^{26}$ M. J. He, H. Qi, Y. T. Ren, Y. J. Zhao, and M. Antezza, "Active control of nearfield radiative heat transfer by coating-twisting method," Opt. Lett. 45, 2914-2917 (2020).

${ }^{27}$ Y. Zhang, H. L. Yi, and H. P. Tan, "Near-field radiative heat transfer between black phosphorus sheets via anisotropic surface plasmon polaritons," ACS Photonics 5, 3739-3747 (2018).

${ }^{28}$ X. L. Liu, J. D. Shen, and Y. M. Xuan, "Pattern-free thermal modulator via thermal radiation between van der Waals materials," J. Quant. Spectrosc. Radiat. Transfer 200, 100-107 (2017).

${ }^{29}$ A. Ghanekar, M. Ricci, Y. P. Tian, O. Gregory, and Y. Zheng, "Strain-induced modulation of near-field radiative transfer," Appl. Phys. Lett. 112, 241104 (2018).

${ }^{30}$ J. L. Song, L. Lu, B. W. Li, B. Zhang, R. Hu, X. P. Zhou, and Q. Cheng, "Thermal routing via near-field radiative heat transfer," Int. J. Heat Mass Transfer 150, 119346 (2020).

${ }^{31}$ P. Ben-Abdallah, "Photon thermal Hall effect," Phys. Rev. Lett. 116, 084301 (2016).

${ }^{32}$ P. Ben-Abdallah, A. Belarouci, L. Frechette, and S.-A. Biehs, "Heat flux splitter for near-field thermal radiation,” Appl. Phys. Lett. 107, 053109 (2015).

${ }^{33}$ S.-A. Biehs, P. Ben-Abdallah, F. S. S. Rosa, K. Joulain, and J.-J. Greffet, "Nanoscale heat flux between nanoporous materials," Opt. Express 19, A1088-A1103 (2011).

${ }^{34}$ R. B. Tao, Z. Chen, and P. Sheng, "First-principles Fourier approach for the calculation of the effective dielectric constant of periodic composites," Phys. Rev. B 41, 2417-2420 (1990).

${ }^{35}$ C. W. Haggans, L. F. Li, and R. K. Kostuk, "Effective-medium theory of zerothorder lamellar gratings in conical mountings," J. Opt. Soc. Am. A 10, 2217-2225 (1993).

${ }^{36}$ M. Antezza, H. B. Chan, B. Guizal, V. N. Marachevsky, R. Messina, and M. K. Wang, "Giant Casimir torque between rotated gratings and the $\theta=0$ anomaly,” Phys. Rev. Lett. 124, 013903 (2020).

${ }^{37}$ P. Ben-Abdallah, S.-A. Biehs, and K. Joulain, "Many-body radiative heat transfer theory,” Phys. Rev. Lett. 107, 114301 (2011).

${ }^{38} \mathrm{P}$. Ben-Abdallah, "Multitip near-field scanning thermal microscopy," Phys. Rev. Lett. 123, 264301 (2019).

${ }^{39}$ J. Dong, J. M. Zhao, and L. H. Liu, "Radiative heat transfer in many-body systems: Coupled electric and magnetic dipole approach,” Phys. Rev. B 95, 12541 (2017).

${ }^{40}$ P. Ben-Abdallah, R. Messina, S.-A. Biehs, M. Tschikin, K. Joulain, and C. Henkel, "Heat superdiffusion in plasmonic nanostructure networks," Phys. Rev. Lett. 111, 174301 (2013). 
${ }^{41}$ E. Tervo, Z. M. Zhang, and B. Cola, "Collective near-field thermal emission from polaritonic nanoparticle arrays,” Phys. Rev. Mater. 1, 015201 (2017).

${ }^{42}$ E. Tervo, M. Francoeur, B. Cola, and Z. M. Zhang, "Thermal radiation in systems of many dipoles," Phys. Rev. B 100, 205422 (2019).

${ }^{43}$ M. G. Luo, J. Dong, J. M. Zhao, L. H. Liu, and M. Antezza, "Radiative heat transfer between metallic nanoparticle clusters in both near field and far field," Phys. Rev. B 99, 134207 (2019).

${ }^{44}$ M. G. Luo, J. M. Zhao, and L. H. Liu, "Near-field radiative heat transfer in a chain of nanoparticles with another chain in proximity," J. Quant. Spectrosc. Radiat. Transfer 243, 106801 (2020).

${ }^{45}$ M. G. Luo, J. M. Zhao, L. H. Liu, and M. Antezza, "Radiative heat transfer and radiative thermal energy for two-dimensional nanoparticle ensembles," Phys. Rev. B 102, 024203 (2020).
${ }^{46}$ E. Palik, Handbook of Optical Constants of Solids (Academic, New York, 1998).

${ }^{47}$ X. L. Liu, R. Zhang, and Z. M. Zhang, "Near-field radiative heat transfer with doped-silicon nanostructured metamaterials," Int. J. Heat Mass Transfer 73, 389-398 (2014).

${ }^{48}$ M. J. He, H. Qi, Y. T. Ren, Y. J. Zhao, and M. Antezza, "Magnetoplasmonic manipulation of nanoscale thermal radiation using twisted graphene gratings," Int. J. Heat Mass Transfer 150, 119305 (2020).

${ }^{49}$ I. M. Fradkin, S. A. Dyakov, and N. A. Gippius, "Fourier modal method for the description of nanoparticle lattices in the dipole approximation," Phys. Rev. B 99, 075310 (2019).

${ }^{50}$ I. M. Fradkin, S. A. Dyakov, and N. A. Gippius, "Nanoparticle lattices with bases: Fourier modal method and dipole approximation," Phys. Rev. B 102, 045432 (2020). 